\title{
New states above charm threshold
}

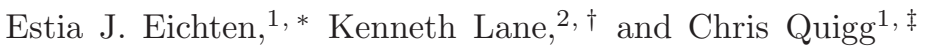 \\ ${ }^{1}$ Theoretical Physics Department \\ Fermi National Accelerator Laboratory \\ P.O. Box 500, Batavia, IL 60510 \\ ${ }^{2}$ Department of Physics, Boston University \\ 590 Commonwealth Avenue, Boston, MA 02215
}

\begin{abstract}
We revise and extend expectations for the properties of charmonium states that lie above charm threshold, in light of new experimental information. We refine the Cornell coupled-channel model for the coupling of $c \bar{c}$ levels to two-meson states, defining resonance masses and widths by pole positions in the complex energy plane, and suggest new targets for experiment.

PACS numbers: 14.40.Gx,13.25.Gv,14.40.Lb
\end{abstract}

\section{INTRODUCTION}

In the short time since the Belle Collaboration reported sighting the "missing" charmonium state $\eta_{c}^{\prime}\left(2^{1} \mathrm{~S}_{0}\right)$ in exclusive $B \rightarrow K K_{S} K^{-} \pi^{+}$decays [1], new states associated with charmonium have appeared in great profusion. Apart from the long-sought $h_{c}\left(1^{1} \mathrm{P}_{1}\right)$, recently observed by the CLEO Collaboration in the decay $\psi(2 \mathrm{~S}) \rightarrow$ $\pi^{0} h_{c} \rightarrow(\gamma \gamma)\left(\gamma \eta_{c}\right)$ [2, 3], all the newly observed states lie near or above charm threshold. The identification of levels above the $D \bar{D}$ flavor threshold has renewed the importance of theoretical studies that couple the $c \bar{c}$ spectrum to charmed-meson-anticharmed-meson channels.

Motivated by the Belle Collaboration's discovery [4] of the narrow state $X(3872) \rightarrow \pi^{+} \pi^{-} J / \psi$, confirmed in short order by the CDF [5], D $\varnothing[\underline{6}]$, and BaBar [7] Experiments, we explored the influence of open-charm channels on charmonium properties, and profiled the $1^{3} \mathrm{D}_{2}$, $1^{3} \mathrm{D}_{3}$, and $2^{1} \mathrm{P}_{1}$ charmonium candidates for $X(3872)$ [8]. We reaffirmed the single-channel potential-model expectation [9, 10] that the favored $1^{3} \mathrm{D}_{2}$ and $1^{3} \mathrm{D}_{3}$ candidates both should have prominent radiative decays, and noted that the $1^{3} \mathrm{D}_{2}$ might be visible in the $D^{0} \bar{D}^{* 0}$ channel, while the dominant decay of the $1^{3} \mathrm{D}_{3}$ state should be into $D \bar{D}$. We proposed that additional discrete charmonium levels were ripe for discovery as narrow resonances of charmed and anticharmed mesons. Barnes, Godfrey, and Swanson have reached similar conclusions using a different model for the coupling to open charm 11].

Experiments have enriched our knowledge of $X(3872)$, but its precise nature remains open to debate. On current evidence, $X(3872)$ is likely to be a $J^{P C}=1^{++}$state. If so, the surviving $2^{3} \mathrm{P}_{1}$ charmonium candidate is problematic, as we discuss in IVB2 Not every state associated with charmonium need be identified as a $c \bar{c}$ state; we will mention below what some of the interlopers might be. An important goal of predicting the properties of char- monium states above flavor threshold is to tag new states that do not fit the template, and so might represent new spectroscopies.

Four other new particles require confirmation, for each has been seen only in a single experiment so far. Belle 12 has reported $Y(3943 \pm 11 \pm 13)$ in the decay $B \rightarrow K \omega J / \psi$; it is a relatively broad state, with total width $\Gamma=87 \pm$ $22 \pm 26 \mathrm{MeV}$. Belle also reports the state $X(3943 \pm 9)$, seen in $e^{+} e^{-} \rightarrow J / \psi+X[13]$. It is observed to decay into $D \bar{D}^{*}$, but not $D \bar{D}$, which suggests an unnatural parity assignment. The total width is $\Gamma<52 \mathrm{MeV}$.

Belle has observed a narrow $(\Gamma \approx 20 \mathrm{MeV})$ state in $\gamma \gamma \rightarrow D \bar{D}$ that they call $Z(3929 \pm 5 \pm 2)$ [14]. The production and decay characteristics are consistent with a $2^{++}$assignment, and this state is a plausible $\chi_{c 2}^{\prime}\left(2^{3} \mathrm{P}_{2}\right)$ candidate. The most recent addition to the collection is $Y(4260)$, a broad $(\approx 88 \mathrm{MeV}) 1^{--}$level seen by BaBar in $e^{+} e^{-} \rightarrow \gamma \pi^{+} \pi^{-} J / \psi$ [15], with supporting evidence from $B \rightarrow K^{-} J / \psi \pi \pi$ [16].

In this Article, we fix the masses of the $3{ }^{1} \mathrm{~S}_{0}$ and $2^{3} \mathrm{P}_{2}$ charmonium states at the positions of $Y(3943)$ and $Z(3931)$, in order to sharpen our expectations for the properties of other states above charm threshold. We also extend our previous calculations to include additional states at higher masses. We highlight the search for narrow structures in charmed meson-anticharmed meson pairs, and remark on analogue states to be expected in the $b \bar{b}$ spectrum.

\section{EXPERIMENTAL STATUS}

Let us briefly summarize what experiment has taught us about the new levels associated with charmonium, and how the experimental facts square with theoretical expectations.

\section{A. Properties of $\eta_{c}^{\prime}$}

*E-mail: eichten@fnal.gov

${ }^{\dagger}$ E-mail: lane@bu.edu

‡E-mail: quigg@fnal.gov
The hyperfine partner of $\psi(2 \mathrm{~S})$ now seems convincingly established, thanks to observations by the Belle [1, 17], 
CLEO [18], and BaBar [19] experiments. The worldaverage values of the mass and width are $M\left(\eta_{c}^{\prime}\right)=3638 \pm$ $5 \mathrm{MeV}$ and $\Gamma\left(\eta_{c}^{\prime}\right)=14 \pm 7 \mathrm{MeV}$ 20].

The proximity of $\psi^{\prime}$ and $\eta_{c}^{\prime}$ to charm threshold means that communication with open-charm channels will alter the properties of these two narrow levels. As we elaborated in Ref. [8], the basic coupled-channel interaction is spin-independent, but the unequal masses and quantum numbers of the $2^{3} \mathrm{~S}_{1}$ and $2^{1} \mathrm{~S}_{0}$ potentialmodel eigenstates mean that their interaction with different charmed-meson-anticharmed-meson channels induces spin-dependent forces that affect the charmonium states. These spin-dependent forces give rise to S-D mixing that contributes to the $\psi(3770)$ electronic width, for example, and are a source of additional spin splitting.

In a single-channel nonrelativistic potential picture, the $2 \mathrm{~S}$ hyperfine splitting is given by $M\left(\psi^{\prime}\right)-M\left(\eta_{c}^{\prime}\right)=$ $32 \pi \alpha_{s}|\Psi(0)|^{2} / 9 m_{c}^{2}$. Normalizing to the observed $1 \mathrm{~S}$ hyperfine splitting, $M(J / \psi)-M\left(\eta_{c}\right)=117 \mathrm{MeV}$, we would find $M\left(\psi^{\prime}\right)-M\left(\eta_{c}^{\prime}\right)=67 \mathrm{MeV}$, to be compared with the observed $48 \pm 5 \mathrm{MeV}$ separation. The $2 \mathrm{~S}$ induced shifts calculated in Ref. 8] draw $\psi^{\prime}$ and $\eta_{c}^{\prime}$ closer by $20.9 \mathrm{MeV}$, substantially improving the agreement between theory and experiment. It is tempting to conclude that the $\psi^{\prime}-\eta_{c}^{\prime}$ splitting reflects the influence of virtual decay channels.

\section{B. CLEO's $h_{c}$ candidate}

Like the $\eta_{c}^{\prime}$, the spin-singlet $L=1$ level of charmonium has been an object of desire for three decades. The barrier to observation has been the absence of allowed E1 or M1 transitions from the $J^{P C}=1^{--}$states readily formed in $e^{+} e^{-}$annihilations. The $1^{1} \mathrm{P}_{1}$ state holds a special interest, beyond the tidiness that would come from completing the spectrum of narrow states. In the nonrelativistic potential-model descriptions that have provided such a reliable guide to quarkonium spectroscopy, the central potential is generally taken to consist of a Coulomb piece with vector Lorentz structure (arising from one-gluon exchange) plus a confining term with scalar Lorentz structure. For such a potential, the hyperfine splitting between spin-singlet and spin-triplet orbital excitations is very small. A significant deviation from the expectation that $M\left(1^{1} \mathrm{P}_{1}\right) \approx\left\langle M\left(1^{3} \mathrm{P}_{J}\right)\right\rangle$ could offer evidence for an unexpected Lorentz structure, or for the importance of effects not included in the nonrelativistic potential-model description.

An earlier observation of $\bar{p} p \rightarrow h_{c} \rightarrow \pi^{0} \eta_{c}$ near the $1^{3} \mathrm{P}_{J}$ centroid in Fermilab Experiment 760 [21] is not sustained by two additional data runs with $2 \times$ and $3 \times$ the luminosity in the successor experiment E835 22, 23]. E835 does report a narrow $(\Gamma<1 \mathrm{MeV})$ event excess in the $\bar{p} p \rightarrow h_{c} \rightarrow \gamma \eta_{c}$ channel at a mass $M\left(h_{c}\right)=3525.8 \pm$ $0.2 \pm 0.2 \mathrm{MeV}$ 22]. The signal bin contains 13 signal events on a 3 -event background, and the signal strength is $\Gamma\left(h_{c} \rightarrow \bar{p} p\right) \mathcal{B}\left(h_{c} \rightarrow \gamma \eta_{c}\right) \approx 11 \mathrm{eV}$.

The CLEO Collaboration has examined 3.08 million $\psi(2 \mathrm{~S})$ decays, in search of examples of the isospinviolating decay to $\pi^{0} h_{c}$, followed by $\pi^{0} \rightarrow \gamma \gamma$ and $h_{c} \rightarrow \gamma \eta_{c}$ 2, 3]. They find $150 \pm 40$ signal counts for the inclusive channel, in which $\eta_{c}$ is not reconstructed, and $17.5 \pm 4.5$ signal counts spread over seven hadronic decay channels of $\eta_{c}$, for a combined significance exceeding $5 \sigma$. In the inclusive sample, the angular distribution of photons in the $h_{c} \rightarrow \gamma \eta_{c}$ cascade follows the $1+\cos ^{2} \theta$ pattern characteristic of E1 emission from a spin-1 state. The CLEO experimenters infer the $1^{1} \mathrm{P}_{1}$ mass $M\left(h_{c}\right)=$ $3524.4 \pm 0.6 \pm 0.4 \mathrm{MeV}$, with a product branching fraction $\mathcal{B}\left(\psi(2 \mathrm{~S}) \rightarrow \pi^{0} h_{c}\right) \mathcal{B}\left(h_{c} \rightarrow \gamma \eta_{c}\right)=(4.0 \pm 0.8 \pm 0.7) \times 10^{-4}$.

The $1{ }^{1} \mathrm{P}_{1}$ level lies $1.0 \pm 0.6 \pm 0.4 \mathrm{MeV}$ below the spintriplet centroid, $\left\langle M\left(1^{3} \mathrm{P}_{J}\right)\right\rangle=3525.36 \pm 0.06 \mathrm{MeV}[20]$, consistent with theoretical expectations.

\section{Rare decays of $\psi(3770)$}

The color-multipole expansion [24, 25, 26] yields symmetry relations for hadronic transition rates between charmonium states, but does not enable calculations of rates from first principles. By the Wigner-Eckart theorem for E1-E1 transitions, all the $1^{3} \mathrm{D}_{J} \rightarrow \pi \pi J / \psi$ rates should be equal (for degenerate ${ }^{3} \mathrm{D}_{J}$ states), so a reliable determination of the rate $\Gamma(\psi(3770) \rightarrow \pi \pi J / \psi)$ is important for anticipating the properties of $\psi_{2}$ and $\psi_{3}$. In our survey of $B$-meson gateways to missing charmonium states [9], we adopted the value $\Gamma\left(1^{3} \mathrm{D}_{1} \rightarrow \pi \pi J / \psi\right) \approx$ $45 \mathrm{keV}$.

The CLEO Collaboration has just reported the first high-significance $(11.6 \sigma)$ observation of the decay $\psi(3770) \rightarrow \pi^{+} \pi^{-} J / \psi$ [27]. Their measured branching fraction, $\mathcal{B}\left(\psi(3770) \rightarrow \pi^{+} \pi^{-} J / \psi\right)=(1.89 \pm 0.20 \pm$ $0.20) \times 10^{-3}$, corresponds to a partial width $\Gamma\left(1^{3} \mathrm{D}_{1} \rightarrow\right.$ $\left.\pi^{+} \pi^{-} J / \psi\right) \approx(45 \pm 9) \mathrm{keV}$. [The CLEO rate is consistent with, but much more precise than, the Beijing Spectrometer (BES) observation 28], $\mathcal{B}\left(\psi(3770) \rightarrow \pi^{+} \pi^{-} J / \psi\right)=$ $(3.4 \pm 1.4 \pm 0.9) \times 10^{-3}$.] A less significant $(3.4 \sigma)$ observation of the decay $\psi(3770) \rightarrow \pi^{0} \pi^{0} \mathrm{~J} / \psi$ is consistent with the expectation that the $\pi^{0} \pi^{0} \mathrm{~J} / \psi$ rate should be half the $\pi^{+} \pi^{-} J / \psi$ rate. Accordingly, we shall take $\Gamma\left(1^{3} \mathrm{D}_{J} \rightarrow \pi \pi J / \psi\right)=(68 \pm 15) \mathrm{keV}$ as an improved estimate of the dipion decay rates of the $\psi_{2}$ and $\psi_{3}$. The increase from our earlier estimate does not alter the expectation that the most prominent decay of the $1^{3} \mathrm{D}_{2}$ state should be $\psi_{2} \rightarrow \gamma \chi_{c 1}$.

CLEO has also searched for rare radiative decays of $\psi(3770)$, reporting the preliminary values $\Gamma(\psi(3770) \rightarrow$ $\left.\gamma \chi_{c 1}\right)=(78 \pm 19) \mathrm{keV}$ and $\Gamma\left(\psi(3770) \rightarrow \gamma \chi_{c 2}\right)<$ $20 \mathrm{keV}[29]$. These observations are in line with expectations $\left(\Gamma\left(1^{3} \mathrm{D}_{1} \rightarrow \gamma \chi_{c 1}\right) \approx 59 \mathrm{keV}\right.$ and $\Gamma\left(1^{3} \mathrm{D}_{1} \rightarrow \gamma \chi_{c 2}\right) \approx$ $4 \mathrm{keV}[8])$, and give us no reason to question the estimated E1 transition rates for the other 1D levels (see (IIID). 


\section{Properties of $X(3782)$}

As the best studied of the new $c \bar{c}$-associated states, $X(3872)$ has been subjected to a broad range of diagnostic tests. Upon discovery, $X(3872)$ seemed a likelythough somewhat heavy - candidate for $\psi_{2}$ (or perhaps $\left.\psi_{3}\right)$, but the expected radiative transitions to $\chi_{c}$ states have never been seen. For a state that lies approximately $50 \mathrm{MeV}$ above charm threshold, the narrow width $(\Gamma(X(3872)<2.3 \mathrm{MeV})[4]$ and the absence of a $D \bar{D}$ signal 30] suggest unnatural parity $P=(-1)^{J+1}$. The BaBar Collaboration has set limits on the existence of a charged partner [31]. In 1.96- TeV $\bar{p} p$ collisions, the production characteristics of $X(3872)$ are similar to those of $\psi(2 \mathrm{~S})$ [6]. CDF has determined the fraction of $X(3872)$ arising from $B$ decays as $16.9 \pm 4.9 \pm 2.0 \%$ [32].

The $\pi \pi$ mass spectrum favors high dipion masses 33. suggesting a $J / \psi \rho$ decay that would be incompatible with the identification of $X(3872) \rightarrow \pi^{+} \pi^{-} J / \psi$ as the strong decay of a pure isoscalar state. See, however, the interesting discussion of alternative interpretations in Ref. 34]. Suzuki has suggested [35] that the $\rho$-like behavior of the $\pi^{+} \pi^{-}$mass spectrum in these decays may result from a dominant decay to $\omega J / \psi$ slightly off mass shell and the small (isospin breaking) $\omega-\rho$ mixing. In this case the $X(3872)$ could be an isoscalar state as expected in a charmonium interpretation.

Observing - or limiting - the $\pi^{0} \pi^{0} \mathrm{~J} / \psi$ decay remains an important goal 10. An observed $J / \psi \pi^{+} \pi^{-} \pi^{0}$ decay suggests an appreciable transition rate to $J / \psi \omega[36]$. Belle's 4.4- $\sigma$ observation of the decay $X(3872) \rightarrow$ $J / \psi \gamma$ [36] determines $C=+$, opposite to the chargeconjugation of the leading charmonium candidates. Finally, an analysis of angular distributions [37] supports the assignment $J^{P C}=1^{++}[38$, but the mass of $X(3872)$ is too low to be gracefully identified with the $2^{3} \mathrm{P}_{1}$ charmonium state, especially if $Z(3931)$ (cf. $\oint$ 【E3) is to be identified as the $2^{3} \mathrm{P}_{2}$ level. The $\chi_{c 1}^{\prime}$ also lacks an isospin-allowed $\pi \pi J / \psi$ decay mode, and acquires a substantial $D \bar{D}^{*}$ width if $M\left(\chi_{c 1}^{\prime}\right)>M\left(D^{0}\right)+M\left(D^{* 0}\right)$. We shall examine this hypothesis further in IVB2

If $X(3872)$ is not a charmonium level, what might it be 39]? Three interpretations take the near-coincidence of the new state's mass and the $D^{0} \bar{D}^{* 0}$ to be a decisive clue: an $s$-wave cusp at $D^{0} \bar{D}^{* 0}$ threshold 40], a $D^{0}-$ $\bar{D}^{* 0}$ "molecule" bound by pion exchange 41, 42, [43, and a diquark-antidiquark "tetraquark" state $[c q][\bar{c} \bar{q}] \underline{44}$, 45. According to a different four-quark interpretation, $X(3872)$ is a $c \bar{c} q \bar{q}$ state organized by the chromomagnetic (color-hyperfine) interaction into a $(c \bar{c})_{\boldsymbol{8}^{-}}(q \bar{q})_{\mathbf{8}}$ pair [46].

What distinctive predictions might allow us to put these interpretations to the test? On the threshold enhancement interpretation, we should expect bumps at many thresholds, but no radial or orbital excitations. If pion exchange is decisive, then there should be no analogue molecule at $D_{s} \bar{D}_{s}^{*}$ threshold. The tetraquark interpretation suggests that $X(3872)$ should be split into two levels, because $[c u][\bar{c} \bar{u}]$ and $[c d][\bar{c} \bar{d}]$ would be displaced by about $7 \mathrm{MeV}$. A first experimental test by the BaBar Collaboration is inconclusive [16]. In $61.2 \pm 15.3$ events that fit the hypothesis $B^{-} \rightarrow K^{-} X(3872)$, they determine a mass of $3871.3 \pm 0.6 \pm 0.1 \mathrm{MeV}$, whereas $8.3 \pm 4.5$ $B^{0} \rightarrow K^{0} X(3872)$ events yield $3868.6 \pm 1.2 \pm 0.2 \mathrm{MeV}$. The mass difference, $2.7 \pm 1.3 \pm 0.2 \mathrm{MeV}$, doesn't yet distinguish between one $X$ and two. If diquarks are useful dynamical objects, there should be a sequence of excited states as well. On the color-hyperfine $c \bar{c} q \bar{q}$ interpretation, the only analogous level should be a $(b \bar{b})_{\mathbf{8}^{-}}(q \bar{q})_{\mathbf{8}}$ state near $B \bar{B}^{*}$ threshold.

What if the $D^{0} \bar{D}^{* 0}$ threshold is not the decisive element? Hybrid $c \bar{c}$-gluon states might appear in the charmonium spectrum 47]. The mass and $1^{++}$quantum numbers of $X(3872)$ do not match lattice-QCD expectations [48, 49, 50]. The valence gluon in the hybrid charmonium wave function leads to speculation that the $\eta J / \psi$ decay mode might be quite prominent. The BaBar experiment [51] has found no sign of $X(3872) \rightarrow \eta J / \psi$. More generally, it is plausible that the gluonic degrees of freedom should manifest themselves as "vibrational states" in the charmonium spectrum 52, 53. While theoretical estimates of the spectrum of string-vibration modes do not reproduce the characteristics of $X(3872)$, we should be alert to the appearance of such states elsewhere.

\section{E. Evidence for states beyond $X(3782)$}

The other new states associated with charmonium require confirmation and elaboration. For the moment, experiments have identified four distinct candidates.

\section{1. $Y(3940)$}

Exploring the $J / \psi \omega$ mass spectrum in decays $B \rightarrow$ $K J / \psi \omega$, the Belle Collaboration observed an enhancement of $58 \pm 11$ events near threshold 12]. Treated as an $s$-wave resonance, this state, named $Y(3940)$, has a mass $M(Y)=3943 \pm 11 \pm 13 \mathrm{MeV}$ and a total width $\Gamma=87 \pm 22 \pm 26 \mathrm{MeV}$. The mass of this object lies well above $D D^{*}$ threshold, so if $Y(3940)$ is a charmonium state, it would be expected to decay dominantly into $D \bar{D}$ or $D \bar{D}^{*}$. A small $J / \psi \omega$ decay rate would complicate the interpretation of the product branching fraction, $\mathcal{B}(B \rightarrow$ $K Y(3940)) \mathcal{B}(Y(3940) \rightarrow J / \psi \omega)=(7.1 \pm 1.3 \pm 3.1) \times 10^{-5}$. For comparison, the branching fractions for the decays $B^{+} \rightarrow K^{+}(c \bar{c})$, with $(c \bar{c})=\eta_{c}, J / \psi, \chi_{c 0}, \chi_{c 1}, \psi^{\prime}$, are between $6-10 \times 10^{-4}[20$. This suggests a branching ratio of about $10 \%$ for $Y(3940) \rightarrow \omega J / \psi$ if $Y(3940)$ is a conventional charmonium state. In contrast, a $c \bar{c}$-gluon hybrid state might decay preferentially into $J / \psi$ or $\psi^{\prime}$ plus light hadrons [54, 55]. To settle the identity of $Y(3940)$ will require confirming that it is a single state and distinguishing it from the $X(3940)$, to which we now turn. 


\section{2. $X(3940)$}

The Belle [17, 56] and BaBar Collaborations [57] have observed copious double charmonium production in $e^{+} e^{-}$ annihilations, with strong signals for $J / \psi \eta_{c}, J / \psi \chi_{c 0}$, and $J / \psi \eta_{c}^{\prime}$. Searching in the region above $D \bar{D}$ threshold, Belle has observed a new peak at $3943 \pm 6 \pm 6 \mathrm{MeV}$, with a width $\Gamma<52 \mathrm{MeV}$ at $90 \%$ C.L. [13].

The new structure, given the temporary name $X(3940)$, is seen to decay into $D \bar{D}^{*}$, with a branching fraction $\mathcal{B}\left(X(3940) \rightarrow D \bar{D}^{*}\right)=0.96_{-0.32}^{+0.45} \pm 0.22$. The absence of any signal in the $D \bar{D}$ channel (for which the branching fraction is $<0.41$ at $90 \%$ C.L.) suggests that $X(3940)$ is an unnatural parity state. Its properties roughly match those expected for $\eta_{c}(3 \mathrm{~S})$, and we shall examine that hypothesis further in

\section{3. $Z(3930)$}

The Belle Collaboration has examined the $D \bar{D}$ invariant-mass distribution in $\gamma \gamma$-fusion events in which the transverse momentum of the $D \bar{D}$ pair is small. A narrow peak of $64 \pm 18$ events appears at $M(D \bar{D})=$ $3939 \pm 5 \pm 2 \mathrm{MeV}$ [14]. The state, provisionally named $Z(3930)$, has a total width $\Gamma(Z(3930))=29 \pm 10 \pm 2 \mathrm{MeV}$, and $\Gamma(Z(3930) \rightarrow \gamma \gamma) \mathcal{B}(Z(3930) \rightarrow D \bar{D})=0.18 \pm 0.05 \pm$ $0.03 \mathrm{keV}$, assuming $J=2$. The (helicity) angular distribution strongly prefers $\sin ^{4} \theta^{*}$, which corresponds to a spin-2 resonance, over the flat angular distribution characteristic of spin-0. Belle accordingly proposes to identify $Z(3930)$ as the $2^{3} \mathrm{P}_{2} \chi_{c 2}^{\prime}$. The measured properties are in reasonable accord with those anticipated in Ref. 8]; we probe the $2^{3} \mathrm{P}_{2}$ assignment further in $1 \mathrm{IVB} 1$

$$
\text { 4. } Y(4260)
$$

In a study of initial-state radiation events, $e^{+} e^{-} \rightarrow$ $(\gamma) \pi^{+} \pi^{-} J / \psi$, the BaBar Collaboration has observed an accumulation of events near $4260 \mathrm{MeV}$ in the $\pi^{+} \pi^{-} J / \psi$ invariant mass distribution [15]. The excess of $125 \pm 23$ events can be characterized as a single $J^{P C}=1^{--}$ resonance with mass $4259 \pm 8_{-6}^{+2} \mathrm{MeV}$ and total width $\Gamma(Y(4260))=88 \pm 23_{-4}^{+6} \mathrm{MeV}$, but a more complicated structure has not been ruled out. It is perhaps significant that $Y(4260)$ lies near the $D_{s}^{*+} \bar{D}_{s}^{*-}$ threshold at $4224 \mathrm{MeV}$. BaBar also reports a $3.1 \sigma$ signal for $Y(4260)$ in exclusive $B^{ \pm} \rightarrow K^{ \pm} \pi^{+} \pi^{-} J / \psi$ decays [16]. An excess of $128 \pm 42$ signal events corresponds to a product branching fraction $\mathcal{B}\left(B^{-} \rightarrow K^{-} Y(4260)\right) \mathcal{B}(Y(4260) \rightarrow$ $\left.\pi^{+} \pi^{-} J / \psi\right)=(2.0 \pm 0.7 \pm 0.2) \times 10^{-5}$.

The new state has variously been interpreted as $\psi(4 \mathrm{~S})$, a $c \bar{c} g$ hybrid state [55, 58, 59], and the radial excitation of a diquark-antidiquark state identified with $X(3872)$ 45]. Curiously, the $\pi^{+} \pi^{-} J / \psi$ enhancement occurs in the neighborhood of a local minimum in the cross section for electron-positron annihilation into hadrons. We shall have more to say about possible interpretations in IVC

\section{STRONG DYNAMICS NEAR THRESHOLD}

Near the threshold for open heavy-flavor pair production, light-quark pairs induce significant nonperturbative contributions to the masses, wave functions, and decay properties of physical $Q \bar{Q}$ states. Lattice QCD calculations, once extended into the flavor-threshold region, should provide firm theoretical predictions. At present, only a phenomenological approach can offer a detailed description of these effects.

The effects of light-quark pairs can be described by coupling the potential model $Q \bar{Q}$ states to nearby physical multibody states. In this threshold picture, the strong interactions are broken into sectors defined by the number of valence quarks. We decompose the full Hamiltonian $\mathcal{H}$ as

$$
\mathcal{H}=\sum_{n} \mathcal{H}_{n}+\mathcal{H}_{I}
$$

where $\mathcal{H}_{n}$ is the Hamiltonian that governs the $(c \bar{c})+n$ light-quark sector and $\mathcal{H}_{I}$ is the interaction that couples the various sectors.

The dynamics of the $Q \bar{Q}$ states (with no valence light quarks, $q$ ) is described by the interaction $\mathcal{H}_{0}$. In principle, excited $Q \bar{Q}$ states with valence or vibrational gluonic degrees of freedom would also be contained in the spectrum of $\mathcal{H}_{0}$. In practice, a simple nonrelativistic potential model is used to determine the properties of the bound states in this sector.

The two-meson sector $Q \bar{q}+q \bar{Q}$ is described by the Hamiltonian $\mathcal{H}_{2}$. As a first approximation, we shall assume $\mathcal{H}_{2}$ to be represented by the low-lying spectrum of two free heavy-light mesons. The physical situation is more complex. At large separation between the two mesons the interactions are dominated by $t$ channel pion exchanges, when they are allowed. For states very near threshold such as $X(3872)$, such pion exchange in attractive channels might have significant effects on properties of the physical states [60]. At somewhat shorter distances, more complicated interactions exist and new bound states might arise, e.g. molecular states 41, 42, 43, 61, 62, 63. Furthermore, at small $Q \bar{Q}$ separation the $Q \bar{Q}+q \bar{q}$ sector may contribute significantly to $\mathcal{H}_{2}$. In particular, as Swanson has emphasized [64], the $\rho J / \psi$ and $\omega J / \psi$ thresholds lie in the $D \bar{D}$ threshold region. The main landmarks throughout the region we consider are shown in Table \

Because current mastery of nonperturbative quantum chromodynamics does not suffice to derive a realistic description of the interactions that link the $Q \bar{Q}$ and $Q \bar{q}+q \bar{Q}$ sectors, we must resort to a phenomenological Ansatz for $\mathcal{H}_{I}$. Following our earlier work 8], we consider here the Cornell coupled-channel $\left(\mathrm{C}^{3}\right)$ model [65, 66, 67] for the 
TABLE I: Thresholds for decay into open charm and nearby hidden-charm thresholds.

\begin{tabular}{cc}
\hline \hline Channel & Threshold Energy $(\mathrm{MeV})$ \\
\hline$D^{0} \bar{D}^{0}$ & 3729.4 \\
$D^{+} D^{-}$ & 3738.8 \\
$D^{0} \bar{D}^{* 0}$ or $D^{* 0} \bar{D}^{0}$ & 3871.2 \\
$\rho^{0} J / \psi$ & 3872.7 \\
$D^{ \pm} D^{* \mp}$ & 3879.5 \\
$\omega^{0} J / \psi$ & 3879.6 \\
$D_{s}^{+} D_{s}^{-}$ & 3936.2 \\
$D^{* 0} \bar{D}^{* 0}$ & 4013.6 \\
$D^{*+} D^{*-}$ & 4020.2 \\
$\eta^{\prime} J / \psi$ & 4054.7 \\
$f^{0} J / \psi$ & $\approx 4077$ \\
$D_{s}^{+} \bar{D}_{s}^{*-}$ or $D_{s}^{*+} \bar{D}_{s}^{-}$ & 4080.0 \\
$a^{0} J / \psi$ & 4081.6 \\
$\varphi^{0} J / \psi$ & 4116.4 \\
$D_{s}^{*+} D_{s}^{*-}$ & 4223.8 \\
$\Lambda_{c} \bar{\Lambda}_{c}$ & 4569.8 \\
\hline \hline
\end{tabular}

creation of $q \bar{q}$ pairs. A brief discussion of various other models for $\mathcal{H}_{I}$ is contained in the Quarkonium Working Group's CERN Yellow Report 68]. The open-charm threshold region occupies our interest in this study. However, analogous effects are present in the $b \bar{b}$ states near $B \bar{B}$ threshold and $c \bar{b}$ states near $D B$ threshold. A detailed comparison of different heavy-quark systems could provide valuable insight into the correct form for the coupling to light-quark pairs.

The $\mathrm{C}^{3}$ formalism generalizes the $c \bar{c}$ model without introducing new parameters, writing the interaction Hamiltonian in second-quantized form as

$$
\mathcal{H}_{I}=\frac{3}{8} \sum_{a=1}^{8} \int: \rho_{a}(\mathbf{r}) V\left(\mathbf{r}-\mathbf{r}^{\prime}\right) \rho_{a}\left(\mathbf{r}^{\prime}\right): d^{3} r d^{3} r^{\prime},
$$

where $V$ is the charmonium potential and $\rho_{a}(\mathbf{r})=$ $\frac{1}{2} \psi^{\dagger}(\mathbf{r}) \lambda_{a} \psi(\mathbf{r})$ is the color current density, with $\psi$ the quark field operator and $\lambda_{a}$ the octet of $\mathrm{SU}(3)$ matrices. To generate the relevant interactions, $\psi$ is expanded in creation and annihilation operators (for charm, up, down, and strange quarks), but transitions from two mesons to three mesons and all transitions that violate the Zweig rule are omitted. It is a good approximation to neglect all effects of the Coulomb piece of the potential in (2). This simple model for the coupling of charmonium to charmedmeson decay channels gives a qualitative understanding of the structures observed above threshold while preserving the successes of the single-channel $c \bar{c}$ analysis below threshold 66, 67].

\section{A. Mass Shifts}

In the presence of coupling to two-light-quark decay channels, the mass $\omega$ of the quarkonium state $\Psi$ is defined by the eigenvalue equation

$$
\left[\mathcal{H}_{0}+\mathcal{H}_{2}+\mathcal{H}_{I}\right] \Psi=\omega \Psi
$$

TABLE II: Charmonium spectrum, including the influence of open-charm channels. All masses are in $\mathrm{MeV}$. The penultimate column holds an estimate of the spin splitting due to tensor and spin-orbit forces in a single-channel potential model. The last column gives the spin splitting induced by communication with open-charm states, for an initially unsplit multiplet.

\begin{tabular}{lcccc}
\hline \hline State & Mass & Centroid & $\begin{array}{c}\text { Splitting } \\
\text { (Potential) }\end{array}$ & $\begin{array}{c}\text { Splitting } \\
\text { (Induced) }\end{array}$ \\
\hline $1^{1} \mathrm{~S}_{0}$ & $2979.9^{a}$ & $3067.6^{b}$ & $-90.5^{e}$ & +2.8 \\
$1^{3} \mathrm{~S}_{1}$ & $3096.9^{a}$ & & $+30.2^{e}$ & -0.9 \\
$1^{3} \mathrm{P}_{0}$ & $3415.3^{a}$ & & $-114.9^{e}$ & +5.9 \\
$1^{3} \mathrm{P}_{1}$ & $3510.5^{a}$ & $3525.3^{c}$ & $-11.6^{e}$ & -2.0 \\
$1^{1} \mathrm{P}_{1}$ & $3524.4^{f}$ & & $+0.6^{e}$ & +0.5 \\
$1^{3} \mathrm{P}_{2}$ & $3556.2^{a}$ & & $+31.9^{e}$ & -0.3 \\
$2^{1} \mathrm{~S}_{0}$ & $3638^{a}$ & $3674^{b}$ & $-50.1^{e}$ & +15.7 \\
$2^{3} \mathrm{~S}_{1}$ & $3686.0^{a}$ & & $+16.7^{e}$ & -5.2 \\
$1^{3} \mathrm{D}_{1}$ & $3769.9^{a}$ & & -40 & -39.9 \\
$1^{3} \mathrm{D}_{2}$ & 3830.6 & $(3815)^{d}$ & 0 & -2.7 \\
$1^{1} \mathrm{D}_{2}$ & 3838.0 & & 0 & +4.2 \\
$1^{3} \mathrm{D}_{3}$ & 3868.3 & & +20 & +19.0 \\
$2^{3} \mathrm{P}_{0}$ & 3881.4 & & -90 & +27.9 \\
$2^{3} \mathrm{P}_{1}$ & 3920.5 & $(3922)^{d}$ & -8 & +6.7 \\
$2^{1} \mathrm{P}_{1}$ & 3919.0 & & 0 & -5.4 \\
$2^{3} \mathrm{P}_{2}$ & $3931^{g}$ & & +25 & -9.6 \\
$3^{1} \mathrm{~S}_{0}$ & $3943^{h}$ & $(4015)^{i}$ & $-66^{e}$ & -3.1 \\
$3^{3} \mathrm{~S}_{1}$ & $4040^{a}$ & & $+22^{e}$ & +1.0 \\
\hline \hline
\end{tabular}

${ }^{a}$ Observed mass, from Review of Particle Physics, Ref. [20].

${ }^{b}$ Input to potential determination.

${ }^{c}$ Observed $1^{3} \mathrm{P}_{J}$ centroid.

${ }^{d}$ Computed centroid.

${ }^{e}$ Required to reproduce observed masses.

${ }^{f}$ Observed mass from CLEO 3 .

${ }^{g}$ Observed mass from Belle 14].

${ }^{h}$ Observed mass from Belle [13].

${ }^{i}$ Observed 3S centroid.

Above the flavor threshold, $\omega$ is a complex eigenvalue.

The basic coupled-channel interaction $\mathcal{H}_{I}$ given by (2) is independent of the heavy quark's spin, but the hyperfine splittings of $D$ and $D^{*}, D_{s}$ and $D_{s}^{*}$, induce spin-dependent forces that affect the charmonium states. These spin-dependent forces give rise to S-D mixing that contributes to the electronic widths of ${ }^{3} \mathrm{D}_{1}$ states and induces additional spin splitting among the physical states.

The masses that result from the full coupled-channel analysis are shown in the second column of Table [II which revises and extends our previously published results [8]. The new version presented here includes the $3 \mathrm{~S}$ levels and takes account of Belle's evidence 14 for $Z$ (3930), interpreted as a $2^{3} \mathrm{P}_{2}$ state (cf. in our earlier analysis, the parameters of the potentialmodel sector governed by $\mathcal{H}_{0}$ must be readjusted to fit the physical masses, $\omega$, to the observed experimental values. The centroids of the $1 \mathrm{D}$ and $2 \mathrm{P}$ spin-triplet masses are pegged to the observed masses of $1^{3} \mathrm{D}_{1}[\psi(3770)]$ and 
$2^{3} \mathrm{P}_{2}[Z(3930)]$, respectively. The assumed spin splittings in the single-channel potential model are shown in the penultimate column and the induced coupled-channel spin splittings for initially unsplit multiplets are presented in the rightmost column of Table [II The shifts induced in the low-lying $1 \mathrm{~S}$ and $1 \mathrm{P}$ levels are small. For all the other states, coupled-channel effects are noticeable and interesting.

An important consequence of coupling to the opencharm threshold is that the $\psi^{\prime}$ receives a downward shift through its communication with the nearby $D \bar{D}$ channel; the unnatural parity $\eta_{c}^{\prime}$ does not couple to $D \bar{D}$, and so is not depressed in the same degree. This effect is implicitly present in the early Cornell papers [66, 67], but the shift of spin-singlet states was not calculated there. The first explicit mention - and the first calculation - of the unequal effects on the masses of the $2 \mathrm{~S}$ hyperfine partners is due to Martin and Richard [69]. In the framework of the $\mathrm{C}^{3}$ model, we found [8, 9] (cf. Table III) that the induced shifts draw $\psi^{\prime}$ and $\eta_{c}^{\prime}$ closer by $20.9 \mathrm{MeV}$, substantially improving the agreement between theory and experiment. This suggests that the $\psi^{\prime}-\eta_{c}^{\prime}$ splitting reflects the influence of virtual decay channels. In the case of the $3 \mathrm{~S}$ system, both the $3^{1} \mathrm{~S}_{0} \eta_{c}^{\prime \prime}$ and the $3^{3} \mathrm{~S}_{1} \psi(4040)$ communicate with open decay channels, and the $\mathrm{C}^{3}$ model leads to a modest $9-\mathrm{MeV}$ increase in the interval between them, as seen in Table

\section{B. Mixing and Properties of Physical States}

The physical states are not pure potential-model eigenstates but include components with two virtual (real, above threshold) open-flavor meson states. Separating the physical state $\Psi$ into $Q \bar{Q}\left(\Psi_{0}\right)$ and two-charmedmeson components $\left(\Psi_{2}\right)$, the resulting decomposition of $\mathcal{H}$ by sector leads to an effective Hamiltonian for the $Q \bar{Q}$ sector given by

$$
\left[\mathcal{H}_{0}+\mathcal{H}_{I}^{\dagger} \frac{1}{\omega-\mathcal{H}_{2}+i \epsilon} \mathcal{H}_{I}\right] \Psi_{0}=\omega \Psi_{0} .
$$

Solving Eqn. (4) in the $Q \bar{Q}$ sector determines the mixing among the potential-model states and the coupling to decay channels. Calculational procedures for the $\mathrm{C}^{3}$ model based on the coupling Hamiltonian (2) have been described in detail elsewhere [8, 66, 67]. Even above threshold for strong decays, the coupled-channel Hamiltonian approach (4) allows a definition of the mass and width of the resonances in terms of the complex eigenvalues and a decomposition of the state into the $c \bar{c}$ and $D \bar{D}$ components via the associated eigenvector. Of course, the width and mass so defined will differ somewhat from the resonance mass and width inferred from the observed enhancement in any particular open-charm-meson-pair channel.

The two-meson contributions to the wave functions of the low-lying $c \bar{c}$ states are shown in Table III for the $\mathrm{C}^{3}$ model. The overall probability for the physical state to
TABLE III: Wave function fractions (in percent) in the $\Psi_{0}$ $(c \bar{c})$ and $\Psi_{2}\left(D^{(*)} \bar{D}^{(*)}\right)$ sectors for near-threshold states.

\begin{tabular}{|c|c|c|c|c|c|}
\hline State & $\begin{array}{c}\Psi_{0} \text { fraction } \\
\mathcal{Z}_{c \bar{c}}\end{array}$ & & $D \bar{D}$ & $\begin{array}{l}\Psi_{2} \text { fraction } \\
D \bar{D}^{*}+D^{*} \bar{D}\end{array}$ & $D^{*} \bar{D}^{*}$ \\
\hline $1^{3} \mathrm{D}_{1}$ & 50.03 & $\begin{array}{l}u \\
d \\
s\end{array}$ & $\begin{array}{c}17.76 \\
15.83 \\
1.33\end{array}$ & $\begin{array}{l}4.43 \\
4.18 \\
0.85\end{array}$ & $\begin{array}{l}2.42 \\
2.38 \\
0.79\end{array}$ \\
\hline $1^{3} \mathrm{D}_{2}$ & 61.10 & $\begin{array}{l}u \\
d \\
s\end{array}$ & $\begin{array}{l}0.00 \\
0.00 \\
0.00\end{array}$ & $\begin{array}{c}15.10 \\
13.94 \\
2.38\end{array}$ & $\begin{array}{l}3.30 \\
3.21 \\
0.96\end{array}$ \\
\hline $1^{1} \mathrm{D}_{2}$ & 63.36 & $\begin{array}{l}u \\
d \\
s\end{array}$ & $\begin{array}{l}0.00 \\
0.00 \\
0.00\end{array}$ & $\begin{array}{c}12.33 \\
11.38 \\
2.00\end{array}$ & $\begin{array}{l}4.88 \\
4.74 \\
1.31 \\
\end{array}$ \\
\hline $1^{3} \mathrm{D}_{3}$ & 50.06 & $\begin{array}{l}u \\
d \\
s\end{array}$ & $\begin{array}{c}16.51 \\
13.17 \\
0.25\end{array}$ & $\begin{array}{l}1.59 \\
1.54 \\
0.43\end{array}$ & $\begin{array}{l}7.47 \\
7.20 \\
1.77 \\
\end{array}$ \\
\hline $2^{3} \mathrm{P}_{0}$ & 50.03 & $\begin{array}{l}d \\
s\end{array}$ & $\begin{array}{c}16.48 \\
17.74 \\
4.41\end{array}$ & $\begin{array}{l}0.00 \\
0.00 \\
0.00\end{array}$ & $\begin{array}{l}5.23 \\
5.04 \\
1.06\end{array}$ \\
\hline $2^{1} \mathrm{P}_{1}$ & 50.04 & $\begin{array}{l}d \\
s\end{array}$ & $\begin{array}{l}0.00 \\
0.00 \\
0.00\end{array}$ & $\begin{array}{c}19.40 \\
18.73 \\
1.43\end{array}$ & $\begin{array}{l}4.95 \\
4.68 \\
0.78\end{array}$ \\
\hline $2^{3} \mathrm{P}_{1}$ & 50.06 & $\begin{array}{l}u \\
d \\
s\end{array}$ & $\begin{array}{l}0.00 \\
0.00 \\
0.00\end{array}$ & $\begin{array}{c}20.82 \\
20.41 \\
2.00\end{array}$ & $\begin{array}{l}3.10 \\
2.99 \\
0.63\end{array}$ \\
\hline $2^{3} \mathrm{P}_{2}$ & 49.95 & $\begin{array}{l}u \\
d \\
s\end{array}$ & $\begin{array}{l}4.47 \\
4.32 \\
0.24\end{array}$ & $\begin{array}{c}12.80 \\
11.47 \\
0.87\end{array}$ & $\begin{array}{l}7.74 \\
7.17 \\
0.97\end{array}$ \\
\hline $3^{1} \mathrm{~S}_{0}$ & 50.05 & $\begin{array}{l}u \\
d \\
s\end{array}$ & $\begin{array}{l}0.00 \\
0.00 \\
0.00\end{array}$ & $\begin{array}{c}20.78 \\
19.82 \\
1.01\end{array}$ & $\begin{array}{l}4.02 \\
3.78 \\
0.55\end{array}$ \\
\hline $3^{3} \mathrm{~S}_{1}$ & 50.04 & $\begin{array}{l}u \\
d \\
s\end{array}$ & $\begin{array}{l}1.42 \\
1.45 \\
0.98\end{array}$ & $\begin{array}{c}10.12 \\
10.24 \\
1.58\end{array}$ & $\begin{array}{c}11.86 \\
11.24 \\
1.08\end{array}$ \\
\hline $2^{3} \mathrm{D}_{1}$ & 50.02 & $s$ & $\begin{array}{l}3.94 \\
3.87 \\
2.69\end{array}$ & $\begin{array}{l}5.34 \\
5.49 \\
3.03\end{array}$ & $\begin{array}{c}12.27 \\
11.89 \\
1.46\end{array}$ \\
\hline
\end{tabular}

be in what we have called the $\Psi_{0}(c \bar{c})$ sector, denoted $\mathcal{Z}_{c \bar{c}}$, decreases as charm threshold is approached. For states above threshold the mixing coefficients become complex, as shown in Table IV Configuration mixing effects modify radiative transition rates [8, 70, 71], in addition to their influence on S-D mixing and spin splittings we have already mentioned.

\section{Zweig-Allowed Strong Decays}

Once the mass of a resonance is given, the $\mathrm{C}^{3}$ formalism yields reasonable predictions for the other resonance properties. We present in Table $\nabla$ our estimates of the strong decay rates for all the $s^{-}, p$-, and $d$-wave charmonium levels that populate the threshold region below $4200 \mathrm{MeV}$, together with what is known from experiment. No new experimental information has come to light about $L \geq 3$ levels; the predictions given in Ref. [8] 
TABLE IV: Charmonium content of states near $c \bar{c}$ threshold. The wave function $\Psi$ reflects mixing induced through communication with open-charm channels. Unmixed potentialmodel eigenstates are denoted by $\left|n^{2 s+1} L_{J}\right\rangle$. The coefficient of the dominant unmixed state is chosen real and positive. Physical states are evaluated at masses given in Table

\begin{aligned} & \hline \hline State Principal $\Psi_{0}(c \bar{c})$ Components \\ & \hline$\Psi\left(1^{3} \mathrm{D}_{1}\right)= 0.10 e^{+0.59 i \pi}|2 \mathrm{~S}\rangle+0.01 e^{+0.81 i \pi}|3 \mathrm{~S}\rangle \\ &+0.69|1 \mathrm{D}\rangle+0.10 e^{+0.86 i \pi}|2 \mathrm{D}\rangle \\ & \Psi\left(1^{3} \mathrm{D}_{2}\right)= 0.77|1 \mathrm{D}\rangle-0.10|2 \mathrm{D}\rangle-0.02|3 \mathrm{D}\rangle \\ & \Psi\left(1^{1} \mathrm{D}_{2}\right)= 0.79|1 \mathrm{D}\rangle-0.10|2 \mathrm{D}\rangle-0.02|3 \mathrm{D}\rangle \\ & \Psi\left(1^{3} \mathrm{D}_{3}\right)= 0.70|1 \mathrm{D}\rangle-0.09|2 \mathrm{D}\rangle+0.02 e^{-0.98 i \pi}|3 \mathrm{D}\rangle \\ & \Psi\left(2^{3} \mathrm{P}_{0}\right)= 0.11 e^{-0.38 i \pi}|1 \mathrm{P}\rangle+0.70|2 \mathrm{P}\rangle+0.03 e^{+0.56 i \pi}|3 \mathrm{P}\rangle \\ & \Psi\left(2^{1} \mathrm{P}_{1}\right)= 0.18 e^{-0.19 i \pi}|1 \mathrm{P}\rangle+0.68|2 \mathrm{P}\rangle+0.07 e^{+0.77 i \pi}|3 \mathrm{P}\rangle \\ & \Psi\left(2^{3} \mathrm{P}_{1}\right)= 0.18 e^{-0.27 i \pi}|1 \mathrm{P}\rangle+0.68|2 \mathrm{P}\rangle+0.07 e^{+0.69 i \pi}|3 \mathrm{P}\rangle \\ & \Psi\left(2^{3} \mathrm{P}_{2}\right)= 0.18 e^{-0.09 i \pi}|1 \mathrm{P}\rangle+0.66|2 \mathrm{P}\rangle+0.07 e^{+0.85 i \pi}|3 \mathrm{P}\rangle \\ & \Psi\left(3^{1} \mathrm{~S}_{0}\right)= 0.02 e^{-0.06 i \pi}|1 \mathrm{~S}\rangle+0.20 e^{-0.18 i \pi}|2 \mathrm{~S}\rangle+0.68|3 \mathrm{~S}\rangle \\ &+0.05 e^{+0.67 i \pi}|4 \mathrm{~S}\rangle \\ & \Psi\left(3^{3} \mathrm{~S}_{1}\right)= 0.02 e^{-0.05 i \pi}|1 \mathrm{~S}\rangle+0.19 e^{-0.30 i \pi}|2 \mathrm{~S}\rangle+0.67|3 \mathrm{~S}\rangle \\ &+0.07 e^{+0.54 i \pi}|4 \mathrm{~S}\rangle \\ &+0.04 e^{+0.59 i \pi}|1 \mathrm{D}\rangle+0.04 e^{+0.59 i \pi}|2 \mathrm{D}\rangle \\ & \Psi\left(2^{3} \mathrm{D}_{1}\right)= 0.02 e^{+0.60 i \pi}|2 \mathrm{~S}\rangle+0.03 e^{-0.71 i \pi}|3 \mathrm{~S}\rangle \\ &+0.14 e^{-0.50 i \pi}|1 \mathrm{D}\rangle+0.69|2 \mathrm{D}\rangle \\ &$\hline \hline\end{aligned}

remain current.

The $1^{3} \mathrm{D}_{1}$ state $\psi^{\prime \prime}(3770)$, which lies some $40 \mathrm{MeV}$ above charm threshold, calibrates the reasonableness of our calculated widths. As we noted in Ref. 8], our value of $20.1 \mathrm{MeV}$ is in excellent agreement with the world average, $\Gamma(\psi(3770))=23.6 \pm 2.7 \mathrm{MeV}[20]$.

The results presented here differ in two respects from those of Ref. [8]. First, the $\chi_{c 2}^{\prime}$ and $\eta_{c}^{\prime \prime}$ masses have been fixed, if only provisionally, by experiment. This results in shifts of the masses and properties of all the $2 \mathrm{P}$ and $3 \mathrm{~S}$ states as shown in Tables $1 \mathrm{D}$ and $\nabla \mathbf{1}$ Second, the present approach allows a more detailed extraction of the composition of the charmonium states above threshold. These results are shown in Tables III and IV

Along with the current PDG values for the total widths of higher $1^{--} c \bar{c}$ resonances, we show in Table $\nabla$ a reanalysis of the existing experimental data by Seth 72 .

The natural-parity $1^{3} \mathrm{D}_{3}$ state can decay into $D \bar{D}$, but its $f$-wave decay is suppressed by the centrifugal barrier factor. Thus the $1^{3} \mathrm{D}_{3}$ may be discovered as a narrow $D \bar{D}$ resonance up to a mass of about $4000 \mathrm{MeV}$.

Barnes, Godfrey, and Swanson [11] have recently reported extensive calculations of decay widths of higher charmonium states in the framework of the ${ }^{3} \mathrm{P}_{0}$ model for quark-pair production. [Shortcomings of both the $\mathrm{C}^{3}$ and ${ }^{3} \mathrm{P}_{0}$ models are assessed in Ref. [68]. Detailed comparisons (e.g. Ackleh, Barnes and Swanson [73]) between various light quark pair creation models are highly desirable.] Unlike the analysis presented here, their calculation does not resum the effects of coupling to decay chan-
TABLE V: Open-charm strong decay modes of the charmonium states near threshold. The theoretical widths using the $\mathrm{C}^{3}$ model [8] are shown.

\begin{tabular}{|c|c|c|c|c|}
\hline \multirow[t]{2}{*}{ State } & \multirow[t]{2}{*}{$n^{2 s+1} L_{J}$} & \multirow[t]{2}{*}{ Mode } & \multicolumn{2}{|c|}{ Decay Width $(\mathrm{MeV})$} \\
\hline & & & Experiment & Computed \\
\hline \multirow[t]{3}{*}{$\psi(3770)$} & \multirow[t]{3}{*}{$1^{3} \mathrm{D}_{1}$} & $D^{0} \bar{D}^{0}$ & & 11.8 \\
\hline & & $D^{+} D^{-}$ & & 8.3 \\
\hline & & total & $23.6 \pm 2.7^{b}$ & 20.1 \\
\hline \multirow[t]{2}{*}{$\psi(3868)$} & \multirow[t]{2}{*}{$1^{3} \mathrm{D}_{3}$} & $D \bar{D}$ & & 0.82 \\
\hline & & total & & 0.82 \\
\hline \multirow[t]{2}{*}{$\chi_{c 0}^{\prime}(3881)$} & \multirow[t]{2}{*}{$2^{3} \mathrm{P}_{0}$} & $D \bar{D}$ & & 61.5 \\
\hline & & total & & 61.5 \\
\hline \multirow{2}{*}{$h_{c 1}^{\prime}(3919)$} & \multirow[t]{2}{*}{$2^{1} \mathrm{P}_{1}$} & $D \bar{D}^{*}$ & & 59.8 \\
\hline & & total & & 59.8 \\
\hline \multirow[t]{2}{*}{$\chi_{c 1}^{\prime}(3920)$} & \multirow[t]{2}{*}{$2^{3} \mathrm{P}_{1}$} & $D \bar{D}^{*}$ & & 81.0 \\
\hline & & total & & 81.0 \\
\hline \multirow[t]{3}{*}{$\chi_{c 2}^{\prime}(3931)$} & \multirow[t]{3}{*}{$2^{3} \mathrm{P}_{2}$} & $D \bar{D}$ & & 21.5 \\
\hline & & $D \bar{D}^{*}$ & & 7.1 \\
\hline & & total & $29 \pm 10($ stat $) \pm 2(\text { sys })^{d}$ & 28.6 \\
\hline \multirow[t]{2}{*}{$\eta_{c}^{\prime \prime}(3943)$} & \multirow[t]{2}{*}{$3^{1} \mathrm{~S}_{0}$} & $D \bar{D}^{*}$ & & 49.8 \\
\hline & & total & $<52^{e}$ & 49.8 \\
\hline \multirow[t]{6}{*}{$\psi(4040)$} & \multirow[t]{6}{*}{$3^{3} \mathrm{~S}_{1}$} & $D \bar{D}$ & & 0.1 \\
\hline & & $D \bar{D}^{*}$ & & 33. \\
\hline & & $D_{s} \bar{D}_{s}$ & & 8. \\
\hline & & $D^{*} \bar{D}^{*}$ & & 33. \\
\hline & & total & $52 \pm 10^{b}$ & 74 \\
\hline & & & $88 \pm 5^{c}$ & 14 \\
\hline \multirow[t]{7}{*}{$\psi(4159)$} & \multirow[t]{7}{*}{$2^{3} \mathrm{D}_{1}$} & $D \bar{D}$ & & 3.2 \\
\hline & & $D \bar{D}^{*}$ & & 6.9 \\
\hline & & $D^{*} \bar{D}^{*}$ & & 41.9 \\
\hline & & $D_{s} \bar{D}_{s}$ & & 5.6 \\
\hline & & $D_{s} \bar{D}_{s}^{*}$ & & 11.0 \\
\hline & & total & $\left.78 \pm 20^{b}\right\}$ & 69.2 \\
\hline & & lotal & $107 \pm 8^{c}$ & 09.2 \\
\hline
\end{tabular}

${ }^{a}$ Computed from CLEO branching fractions [13].

${ }^{b}$ Review of Particle Physics [20].

${ }^{c}$ Reanalysis by Seth 72].

${ }^{d}$ Belle 14.

${ }^{e}$ Belle [13].

nels. The general scale of resulting decay rates is similar to those displayed in Table $\nabla$ For example, Barnes et al. determine $\Gamma\left(3^{3} \mathrm{~S}_{1}\right)=80 \mathrm{MeV}$ and $\Gamma\left(2^{3} \mathrm{D}_{1}\right)=74 \mathrm{MeV}$.

\section{Radiative Transitions}

As Tables III and IV show, the physical charmonium states are not pure potential-model eigenstates. To compute the E1 radiative transition rates, we must take into account both the standard $(c \bar{c}) \rightarrow(c \bar{c}) \gamma$ transitions and the transitions between (virtual) decay channels in the initial and final states. Details of the calculational procedure are given in $\S I V . B$ of Ref. 67]. There we also illuminated the differences between single-channel and 
TABLE VI: Calculated and observed rates for $\mathrm{E} 1$ radiative transitions among charmonium levels. $\mathrm{C}^{3}$ model rates include the influence of open-charm channels. Photon energies, in $\mathrm{MeV}$, in parentheses.

\begin{tabular}{|c|c|c|c|}
\hline & \multicolumn{3}{|c|}{ Partial Width (keV) } \\
\hline $\begin{array}{l}1^{3} \mathrm{D}_{1}(3770) \rightarrow \\
\text { model } \\
\text { CLEO [74] }\end{array}$ & $\begin{array}{c}\chi_{c 2} \gamma(208) \\
3.9 \\
<40\end{array}$ & $\begin{array}{c}\chi_{c 1} \gamma(251) \\
59 \\
75 \pm 18\end{array}$ & $\begin{array}{c}\chi_{c 0} \gamma(338) \\
225 \\
<1100\end{array}$ \\
\hline $\begin{array}{l}1^{3} \mathrm{D}_{2}(3831) \rightarrow \\
\text { model }\end{array}$ & $\begin{array}{c}\chi_{c 2} \gamma(266) \\
45\end{array}$ & $\begin{array}{c}\chi_{c 1} \gamma(308) \\
212\end{array}$ & \\
\hline $\begin{array}{l}1^{3} \mathrm{D}_{3}(3868) \rightarrow \\
\text { model }\end{array}$ & $\begin{array}{c}\chi_{c 2} \gamma(303) \\
286\end{array}$ & & \\
\hline $\begin{array}{l}2^{3} \mathrm{P}_{0}(3881) \rightarrow \\
\text { model }\end{array}$ & $\begin{array}{c}J / \psi \gamma(704) \\
39\end{array}$ & $\begin{array}{c}\psi^{\prime} \gamma(190) \\
17\end{array}$ & $\begin{array}{c}1^{3} \mathrm{D}_{1} \gamma(110) \\
1.6\end{array}$ \\
\hline $\begin{array}{l}2^{3} \mathrm{P}_{1}(3920) \rightarrow \\
\text { model }\end{array}$ & $\begin{array}{c}1^{3} \mathrm{D}_{1} \gamma(147) \\
5.2\end{array}$ & $\begin{array}{c}1^{3} \mathrm{D}_{2} \gamma(86) \\
2.6\end{array}$ & \\
\hline $\begin{array}{l}2^{3} \mathrm{P}_{1}(3920) \rightarrow \\
\text { model }\end{array}$ & $\begin{array}{c}J / \psi \gamma(737) \\
15\end{array}$ & $\begin{array}{c}\psi^{\prime} \gamma(227) \\
75\end{array}$ & \\
\hline $\begin{array}{l}2^{3} \mathrm{P}_{2}(3931) \rightarrow \\
\text { model }\end{array}$ & $\begin{array}{c}1^{3} \mathrm{D}_{1} \gamma(157) \\
0.4\end{array}$ & $\begin{array}{c}1^{3} \mathrm{D}_{2} \gamma(95) \\
3.0\end{array}$ & $\begin{array}{c}1^{3} \mathrm{D}_{3} \gamma(62) \\
3.6\end{array}$ \\
\hline $\begin{array}{l}2^{3} \mathrm{P}_{2}(3931) \rightarrow \\
\text { model }\end{array}$ & $\begin{array}{c}J / \psi \gamma(775) \\
14\end{array}$ & $\begin{array}{c}\psi^{\prime} \gamma(272) \\
120\end{array}$ & \\
\hline $\begin{array}{l}\psi(4040) \rightarrow \\
\text { model }\end{array}$ & $\begin{array}{c}2^{3} \mathrm{P}_{2} \gamma(84) \\
18\end{array}$ & $\begin{array}{c}2^{3} \mathrm{P}_{1} \gamma(132) \\
5.4\end{array}$ & $\begin{array}{c}2^{3} \mathrm{P}_{0} \gamma(156) \\
20\end{array}$ \\
\hline $\begin{array}{l}\psi(4040) \rightarrow \\
\text { model }\end{array}$ & $\begin{array}{c}1^{3} \mathrm{P}_{2} \gamma(456) \\
12\end{array}$ & $\begin{array}{c}1^{3} \mathrm{P}_{1} \gamma(495) \\
0.4\end{array}$ & $\begin{array}{c}1^{3} \mathrm{P}_{0} \gamma(577) \\
0.03\end{array}$ \\
\hline
\end{tabular}

coupled-channel expectations for the radiative rates.

Our expectations for E1 transition rates among spintriplet levels are shown in Table VI Again the observed properties of $\psi(3770)$ confirm the reasonableness of the $\mathrm{C}^{3}$ framework. The radiative decay rates for the $2^{3} \mathrm{P}_{J}$ and $3^{3} \mathrm{~S}_{1}$ levels are calculated at the predicted masses. All these rates are small compared to the expected opencharm decay rates.

\section{IMPLICATIONS FOR NEWFOUND STATES}

\section{A. $X(3940)$ as $\eta_{c}(3 \mathrm{~S})$}

The observation by Belle [13] of a state $X(3940)$ recoiling against the $J / \psi$ system in continuum production at the $\Upsilon(4 \mathrm{~S})$ region fits the general behavior expected for the $\eta_{c}^{\prime \prime}$ charmonium level. This state is not seen to decay into $D \bar{D}$ but does decay into $D \bar{D}^{*}$, suggesting that it has unnatural parity. Figure 1 shows the complex pole position of the $\eta_{c}^{\prime \prime}$ in the $\mathrm{C}^{3}$ model as its bare mass is varied in $10-\mathrm{MeV}$ steps. At $3943 \mathrm{MeV}$, we estimate a $D \bar{D}^{*}$ width of $50 \mathrm{MeV}$, which is quite close to the experimental upper bound on the total width, $\Gamma(X(3940))<52 \mathrm{MeV}$.

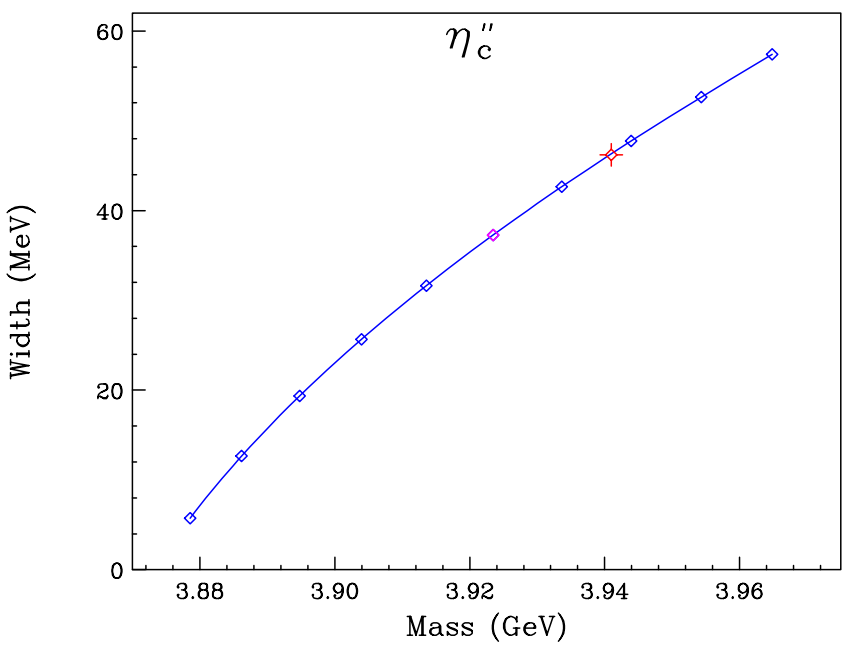

FIG. 1: Variation of the $\eta_{c}^{\prime \prime}$ width with mass in the $\mathrm{C}^{3}$ model. The physical mass of the $X(3940)$ is denoted by a crossed dot.

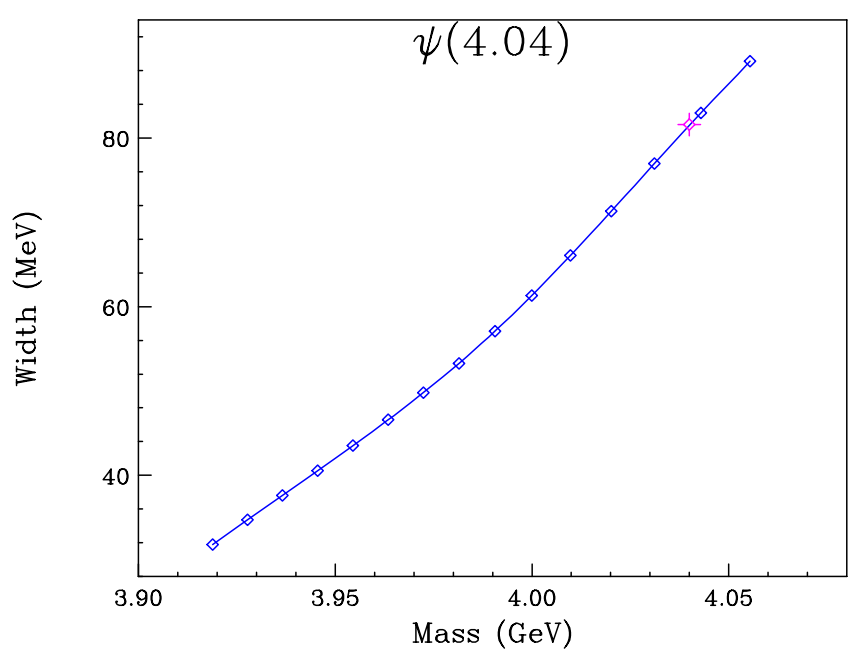

FIG. 2: Width of the $3^{3} \mathrm{~S}_{1}$ state as a function of its mass. The physical mass of the $\psi(4040)$ is denoted by a crossed dot.

If $\psi(4040)$ is assigned to the $3{ }^{3} \mathrm{~S}_{1}$ level, it is somewhat problematic to identify $X(3940)$ as its hyperfine partner. We show in Figure 2 the behavior of the physical mass and width of the triplet $3 \mathrm{~S}$ state as the bare mass is varied in $10-\mathrm{MeV}$ steps. To reproduce the observed masses, we would require the spin splitting in the charmonium sector to be $88 \mathrm{MeV}$, which is considerably larger than the $2 \mathrm{~S}$ splitting, and larger than expected in naive potential models.

If, for the moment, we take the existence, mass, and unnatural parity of $X(3940)$ as well established, then two possible sources of the discrepancy deserve futher investigation.

First, it is difficult to determine the true pole position for the $3{ }^{3} \mathrm{~S}_{1}$ state from measurements of the step in $R_{c \bar{c}} \equiv \sigma\left(e^{+} e^{-} \rightarrow\right.$ hadrons $) / \sigma\left(e^{+} e^{-} \rightarrow \mu^{+} \mu^{-}\right)$[2]. The resonance decay widths are determined from fitting measurements of $\Delta R$ in $e^{+} e^{-}$annihilation to a model 
for each resonance including radiative corrections. This whole procedure is complicated by its dependence on the resonance shape, i.e., the expected non-Breit-Wigner nature of the partial widths for radially excited resonances. It may be more useful to produce a model of $\Delta R$ for direct comparison with data. Greater resolving power between models is possible if the contribution from each individual open heavy flavor final state is separately reported.

For the $\mathrm{C}^{3}$ model, the structure of $\Delta R(c \bar{c})$ in the threshold region was studied in the original Cornell group works [65, 66, 67] and later extended to the $\Delta R(b \bar{b})$ in the threshold region [75]. The structure of $\Delta R(c \bar{c})$ and $\Delta R(b \bar{b})$ has also been studied in ${ }^{3} \mathrm{P}_{0}$ models of quark pair creation 76]. There are also some attempts to compare the different models 77,78$]$.

Second, thresholds corresponding to an $s$-wave plus a $p$-wave charmed meson are nearby. Unlike the two-swave charm meson channels, which are $p$-wave decays for the $3 \mathrm{~S}$ states, these are $s$-wave decays and thus have stronger threshold effects. In particular the lowest mass channel $D\left(J=0 ; j_{l}^{P}=1 / 2^{-}\right) D\left(J=0 ; j_{l}^{P}=1 / 2^{+}\right)$couples to the $3{ }^{1} S_{1} \eta_{c}^{\prime \prime}$ state but not the $3^{3} S_{1} \psi(4040)$ state. This will increase the induced spin-splitting (see Table column 5) between the states. Thus the observed $\psi^{\prime \prime}-\eta_{c}^{\prime \prime}$ separation will correspond to a smaller potential-model splitting (column 4).

\section{B. The $2 \mathrm{P}$ states \\ 1. $Z(3930)$ as $\chi_{c 2}^{\prime}$}

Belle has also observed the new state $Z(3930)$ in twophoton production of $D \bar{D}$ pairs [14]. The $2^{3} \mathrm{P}_{2}$ and $2^{3} \mathrm{P}_{0}$ levels are natural charmonium candidates; experiment favors the $J=2$ assignment. In constructing Table adjusted the $2 \mathrm{P}$ centroid to give the mass of the $J=2$ state in agreement with observation. At the observed mass, we compute $\Gamma\left(2^{3} \mathrm{P}_{2} \rightarrow D \bar{D}\right)=21.5 \mathrm{MeV}$ and $\Gamma\left(2^{3} \mathrm{P}_{2} \rightarrow D \bar{D}^{*}\right)=7.1 \mathrm{MeV}$, in reasonable agreement with experiment (see Table W) We show in Figure 3 the variation of the $2^{3} \mathrm{P}_{2}$ pole position with bare mass in $10-\mathrm{MeV}$ steps, using Eqn. (4) to fully account for finitewidth effects.

\section{2. $X(3872)$ as $2^{3} P_{1}$}

If $X(3872)$ is a $1^{++}$state, the unique surviving $c \bar{c}$ candidate would be the $2^{3} \mathrm{P}_{1}$ level 35. One problem with this interpretation is clear: the expected mass (cf. TableII) is $48 \mathrm{MeV}$ above the observed $X$ mass. If $Z(3930)$ is indeed the $2{ }^{3} \mathrm{P}_{2}$ level, it is very unlikely that its $J=1$ partner should lie at $3872 \mathrm{MeV}$.

If the mass were not a problem, the $2^{3} \mathrm{P}_{1}$ interpretation would still be difficult to sustain. The E1 radiative transition to $\gamma J / \psi$ is greatly suppressed because of a cancel-



FIG. 3: Mass dependence of $\Gamma\left(2^{3} \mathrm{P}_{2}\right)$.

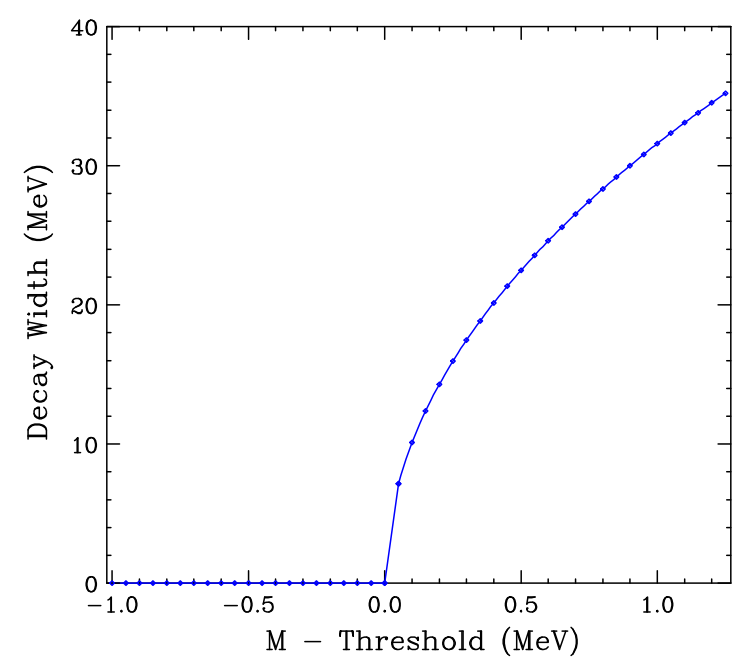

FIG. 4: Mass dependence of $\Gamma\left(2^{3} \mathrm{P}_{1}\right) \rightarrow D \bar{D}^{*}$.

lation between the $c \bar{c}$ and $D \bar{D}^{*}$ contributions. We obtain 35 and $21 \mathrm{keV}$ for the transitions to $J / \psi$ and $\psi^{\prime}$ respectively from the $c \bar{c}$ contributions alone. When the effects of the charmed meson virtual channels are included, the rates change dramatically to 2.4 and $90 \mathrm{keV}$, respectively. This would make the reported observation of the $\gamma J / \psi$ mode highly unlikely. Of course, within the $\mathrm{C}^{3}$ model the isospin breaking between $D^{0} \bar{D}^{* 0}$ and $D^{+} D^{*-}$ virtual states is relatively small. Even if we assume it to be large, so that only the virtual $D^{0} \bar{D}^{* 0}$ component is important, our analysis suggests that a search for the radiative decay to $\psi^{\prime}$ would be valuable.

Finally, the decay rate of $2^{3} \mathrm{P}_{1} \rightarrow D \bar{D}^{*}$ increases very rapidly above threshold as shown in Figure 4 Since the total width of $X(3872)$ is less than $2.3 \mathrm{MeV}$, the possibility that the $2^{3} \mathrm{P}_{1}$ level lies more than $22 \mathrm{keV}(!)$ above the $D^{0} D^{* 0}$ threshold is precluded. When the additional partial width $\Gamma_{\text {other }}$ of the $2^{3} \mathrm{P}_{1}$ into other modes ( $g g g$, $\gamma J / \psi$, etc.) is taken into account, the largest tenable mass 
is lowered to $M\left(D^{0}\right)+M\left(D^{* 0}\right)-\Gamma_{\text {other }}$. Combining this result with the large ratio of $\Gamma\left(X(3872) \rightarrow D^{0} \pi^{0} \bar{D}^{0}\right) \approx$ $10 \Gamma(X(3872) \rightarrow \pi \pi J / \psi)$ reported by Belle 79 renders the $2^{3} \mathrm{P}_{1}$ interpretation very improbable.

\section{C. $Y(4260)$ and other states}

In initial-state radiation events, BaBar 15 has recently reported a $\pi \pi J / \psi$ resonance, $Y(4260)$, with a width of approximately $88 \mathrm{MeV}$. This state is hard to assign within the $c \bar{c}$ spectrum. To be directly produced from a virtual photon, it must be a $J^{P C}=1^{--} \mathrm{S}$ or $\mathrm{D}$ state. The small contribution to $\Delta R$ discourages the $4 \mathrm{~S}$ interpretation, but leaves open the $2^{3} \mathrm{D}_{1}$ identification. However the $\mathrm{C}^{3}$ analysis requires that state at $4.16 \mathrm{GeV}$ in order to reproduce the structure of $\Delta R$. The identification of the $Y(4260)$ with the $2^{3} \mathrm{D}_{1}$ state is ruled out in the $\mathrm{C}^{3}$ model because the partial decay width of the $2 \mathrm{D}$ state (at $4260 \mathrm{MeV}$ ) into charm meson pairs is $125 \mathrm{MeV}$. This is not the discovery mode for $Y(4260)$ but exceeds the reported total width. Therefore, this state does not have a conventional charmonium interpertation. One plausible explanation for this state is that it is a hybrid state [55, 58]. The lattice calculations of Juge and collaborators [48, 50] for the $Q \bar{Q}$ potentials with excited gluonic modes give a lowest multiplet $\left(H_{1}\right.$ in their notation) consisting of a quark spin-singlet $1^{--}$state and a spin-triplet set of states $\left(0^{-+}, 1^{-+}\right.$, and $\left.2^{-+}\right)$. These states would be degenerate in the absence of relativistic spin-dependent mass corrections. If this interpretation were correct, then analogous narrow states would be expected in the $b \bar{b}$ system.

\section{SUMMARY}

The computed properties of the $2^{3} \mathrm{P}_{2}$ and $3{ }^{1} \mathrm{~S}_{0} c \bar{c}$ levels are in rough agreement with the $Z(3931)$ and $X(3943)$ states observed at Belle. We await more statistics and confirmation of these results to make a definitive statement on the identification of these states.

It is extremely important to solidify what appears to be known about $X(3872)$, the best studied of the new states, beginning with its $1^{++}$quantum numbers. We emphasize the need to confirm the $\gamma J / \psi$ decay mode, which fixes the $C=+$ assignment, and to determine the $X(3872) \rightarrow$ $\pi^{0} \pi^{0} \mathrm{~J} / \psi$ branching fraction.

Experiments should continue to search for additional narrow charmonium states in neutral combinations of charmed mesons and anticharmed mesons. The most likely candidates correspond to the $1^{3} \mathrm{D}_{3}, 1^{3} \mathrm{D}_{2}$, and $1^{3} \mathrm{~F}_{4}$ levels [8]. The analysis we have carried out can be extended to the $b \bar{b}$ system, where it may be possible to see discrete threshold-region states as well. For any of these new states that are threshold bound states their mass is primarily determined by the positions of open flavor channels. For example, if the $X(3872)$ arises through additional interaction in the two-meson sector, then in the $b \bar{b}$ system we would expect an analog state not far below $B^{*} \bar{B}$ threshold.

\section{Acknowledgments}

We thank our experimental colleagues for stimulating discussions. KL's research was supported by the Department of Energy under Grant No. DE-FG02-91ER40676. Fermilab is operated by Universities Research Association Inc. under Contract No. DE-AC02-76CH03000 with the U.S. Department of Energy.
[1] S. K. Choi et al. [BELLE collaboration], Phys. Rev. Lett. 89, 102001 (2002) [Erratum-ibid. 89, 129901 (2002)] arXiv:hep-ex/0206002.

[2] J. L. Rosner et al. [CLEO Collaboration], Phys. Rev. Lett. 95, 102003 (2005) arXiv:hep-ex/0505073.

[3] P. Rubin [CLEO Collaboration], "Observation of the ${ }^{1} \mathrm{P}_{1}$ State of Charmonium," arXiv:hep-ex/0508037

[4] S. K. Choi et al. [Belle Collaboration], Phys. Rev. Lett. 91, 262001 (2003) arXiv:hep-ex/0309032.

[5] D. Acosta et al. [CDF II Collaboration], Phys. Rev. Lett. 93, 072001 (2004) arXiv:hep-ex/0312021.

[6] V. M. Abazov et al. [D0 Collaboration], Phys. Rev. Lett. 93, 162002 (2004) arXiv:hep-ex/0405004.

[7] B. Aubert et al. [BABAR Collaboration], Phys. Rev. D 71, 071103 (2005) arXiv:hep-ex/0406022.

[8] E. J. Eichten, K. Lane and C. Quigg, Phys. Rev. D 69, 094019 (2004) arXiv:hep-ph/0401210.
[9] E. J. Eichten, K. Lane and C. Quigg, Phys. Rev. Lett. 89, 162002 (2002) arXiv:hep-ph/0206018.

[10] T. Barnes and S. Godfrey, Phys. Rev. D 69, 054008 (2004) arXiv:hep-ph/0311162.

[11] T. Barnes, S. Godfrey and E. S. Swanson, Phys. Rev. D 72, 054026 (2005) arXiv:hep-ph/0505002.

[12] K. Abe et al. [Belle Collaboration], Phys. Rev. Lett. 94, 182002 (2005) arXiv:hep-ex/0408126.

[13] K. Abe, et al. [Belle Collaboration], "Observation of a new charmonium state in double charmonium production in $e^{+} e^{-}$annihilation at $\sqrt{s} \approx 10.6 \mathrm{GeV}$," arXiv:hep-ex/0507019

[14] S. Uehara, et al. [Belle Collaboration], "Observation of a $\chi_{c 2}^{\prime}$ candidate in $\gamma \gamma \rightarrow D \bar{D}$ production at Belle," arXiv:hep-ex/0512035

[15] B. Aubert et al. [BABAR Collaboration], Phys. Rev. Lett. 95, 142001 (2005) arXiv:hep-ex/0506081. 
[16] B. Aubert et al. [BABAR Collaboration], "Study of $J / \psi \pi^{+} \pi^{-}$states produced in $B^{0} \rightarrow J / \psi \pi^{+} \pi^{-} K^{0}$ and $B^{-} \rightarrow J / \psi \pi^{+} \pi^{-} K^{-}, "$ arXiv:hep-ex/0507090

[17] K. Abe et al. [Belle Collaboration], Phys. Rev. D 70, 071102 (2004) arXiv:hep-ex/0407009.

[18] D. M. Asner et al. [CLEO Collaboration], Phys. Rev. Lett. 92, 142001 (2004) arXiv:hep-ex/0312058.

[19] B. Aubert et al. [BABAR Collaboration], Phys. Rev. Lett. 92, 142002 (2004) arXiv:hep-ex/0311038.

[20] S. Eidelman et al., Phys. Lett. B 592, 1 (2004) and 2005 partial update for the 2006 edition available at http://pdg.lbl.gov/

[21] T. A. Armstrong et al., Phys. Rev. Lett. 69, 2337 (1992).

[22] M. Andreotti et al. [Fermilab E835 Collaboration], Phys. Rev. D 72, 032001 (2005).

[23] D. N. Joffe, "A search for the singlet-P state $h_{c}\left(1^{1} \mathrm{P}_{1}\right)$ of charmonium in proton antiproton annihilations at Fermilab experiment E835," arXiv:hep-ex/0505007

[24] K. Gottfried, Phys. Rev. Lett. 40, 598 (1978).

[25] M. B. Voloshin, Nucl. Phys. B 154, 365 (1979).

[26] T. M. Yan, Phys. Rev. D 22, 1652 (1980).

[27] N. E. Adam [CLEO Collaboration], "Observation of $\psi(3770) \rightarrow \pi \pi J / \psi$ and measurement of $\Gamma_{e e}(\psi(2 \mathrm{~S})), "$ arXiv:hep-ex/0508023

[28] J. Z. Bai et al. [BES Collaboration], Phys. Lett. B 605, 63 (2005) arXiv:hep-ex/0307028.

[29] D. H. Miller, "Recent Charmonium results from CLEO," talk at the Europhysics Conference on High-Energy Particle Physics, Lisbon, 21-27 July 2005.

[30] K. Abe et al. [Belle Collaboration], Phys. Rev. Lett. 93, 051803 (2004) arXiv:hep-ex/0307061.

[31] B. Aubert et al. [BABAR Collaboration], "Search for an $X(3872)$ charged partner in the decay mode $X^{-} \rightarrow$ $J / \psi \pi^{-} \pi^{0}$ in the $B$ meson decays $B^{0} \rightarrow X^{-} K^{+}$and $B^{-} \rightarrow X^{-} K_{S}^{0}$," arXiv:hep-ex/0408083

[32] The CDF II Collaboration, "The 'Lifetime' Distribution of $X(3872)$ Mesons Produced in $\bar{p} p$ Collisions at CDF," CDF Note 7159 .

[33] CDF Collaboration, "Measurement of the dipion mass spectrum in $X(3872) \rightarrow$ $\pi^{+} \pi^{-} J / \psi$ decays," CDF Note 05-03-24, [34] G. Bauer, "The $X(3872)$ at the Tevatron," talk at PANIC05, Santa Fe, http://www .panic05.lanl.gov/abstracts/137/bauer_137
M. Suzuki, "The $X(3872)$ boson: Molecule or charmonium," arXiv:hep-ph/0508258

[36] K. Abe et al., "Evidence for $X(3872) \rightarrow \gamma J / \psi$ and the sub-threshold decay $X(3872) \rightarrow \omega J / \psi, "$ arXiv:hep-ex/0505037

[37] J. L. Rosner, Phys. Rev. D 70, 094023 (2004) arXiv:hep-ph/0408334.

[38] K. Abe et al., "Experimental constraints on the possible $J^{P C}$ quantum numbers of the $X(3872)$," arXiv:hep-ex/0505038

[39] E. Swanson, "Review of Heavy Hadron Spectroscopy," arXiv:hep-ph/0509327

[40] D. V. Bugg, Phys. Lett. B 598, 8 (2004) arXiv:hep-ph/0406293; Phys. Rev. D 71, 016006 (2005) arXiv:hep-ph/0410168. See also Phys. Rept. 397, 257 (2004) arXiv:hep-ex/0412045.

[41] N. A. Tørnquist Phys. Lett. B590, 209 (2004) arXiv:hep-ph/0402237.

[42] E. S. Swanson, J. Phys.: Conf. Ser. 9, 79-82 (2005).
[43] M. B. Voloshin, Phys. Lett. B 579, 316 (2004) arXiv:hep-ph/0309307.

[44] L. Maiani, F. Piccinini, A. D. Polosa and V. Riquer, Phys. Rev. D 71, 014028 (2005) arXiv:hep-ph/0412098.

[45] L. Maiani, V. Riquer, F. Piccinini and A. D. Polosa, Phys. Rev. D 72, 031502 (2005) arXiv:hep-ph/0507062.

[46] H. Hogaasen, J. M. Richard and P. Sorba, "A chromomagnetic mechanism for the $\mathrm{X}(3872)$ resonance," arXiv:hep-ph/0511039

[47] F. E. Close and S. Godfrey, Phys. Lett. B 574, 210 (2003) arXiv:hep-ph/0305285.

[48] K. J. Juge, J. Kuti and C. J. Morningstar, Phys. Rev. Lett. 82, 4400 (1999) arXiv:hep-ph/9902336.

[49] X. Liao and T. Manke, "Excited charmonium spectrum from anisotropic lattices," arXiv:hep-lat/0210030

[50] K. J. Juge, J. Kuti and C. Morningstar, Phys. Rev. Lett. 90, 161601 (2003) arXiv:hep-lat/0207004.

[51] B. Aubert et al. [BABAR Collaboration], Phys. Rev. Lett. 93, 041801 (2004) arXiv:hep-ex/0402025.

[52] R. Giles and S. H. H. Tye, Phys. Rev. D 16, 1079 (1977).

[53] W. Buchmuller and S. H. H. Tye, Phys. Rev. Lett. 44, 850 (1980).

[54] F. E. Close, Phys. Lett. B 342, 369 (1995) arXiv:hep-ph/9409203.

[55] F. E. Close and P. R. Page, "Gluonic charmonium resonances at BaBar and Belle?" arXiv:hep-ph/0507199

[56] K. Abe et al. [Belle Collaboration], Phys. Rev. Lett. 89, 142001 (2002) arXiv:hep-ex/0205104.

[57] B. Aubert et al. [BABAR Collaboration], Phys. Rev. D 72, 031101 (2005) arXiv:hep-ex/0506062.

[58] S. L. Zhu, Phys. Lett. B 625, 212 (2005) arXiv:hep-ph/0507025.

[59] E. Kou and O. Pène, "Suppressed decay into open charm for the $Y(4260)$ being an hybrid," arXiv:hep-ph/0507119

[60] E. Braaten and M. Kusunoki, Phys. Rev. D 69, 074005 (2004) arXiv:hep-ph/0311147.

[61] M. B. Voloshin and L. B. Okun, JETP Lett. 23, 333 (1976) [Pisma Zh. Eksp. Teor. Fiz. 23, 369 (1976)].

[62] A. De Rujula, H. Georgi and S. L. Glashow, Phys. Rev. Lett. 38, 317 (1977).

[63] A. De Rujula and R. L. Jaffe, "Quark Chemistry: Charhxmilifipi.\$\$lecules," in Experimental meson spectroscopy 1977, edited by E. von Goeler and R. Weinstein (Northeastern University Press, Boston, 1977), p. 83.

触iE05.S.dfíwanson, Phys. Lett. B 588, 189 (2004) arXiv:hep-ph/0311229.

[65] E. Eichten, K. Gottfried, T. Kinoshita, K. D. Lane and T. M. Yan, Phys. Rev. Lett. 36, 500 (1976).

[66] E. Eichten, K. Gottfried, T. Kinoshita, K. D. Lane and T. M. Yan, Phys. Rev. D 17, 3090 (1978) [Erratum-ibid. D 21, 313 (1980)].

[67] E. Eichten, K. Gottfried, T. Kinoshita, K. D. Lane and T. M. Yan, Phys. Rev. D 21, 203 (1980).

[68] N. Brambilla et al., "Heavy quarkonium physics," arXiv:hep-ph/0412158

[69] A. Martin and J. M. Richard, Phys. Lett. B 115, 323 (1982).

[70] J. L. Rosner, Phys. Rev. D 64, 094002 (2001) arXiv:hep-ph/0105327.

[71] J. L. Rosner, Annals Phys. 319, 1 arXiv:hep-ph/0411003.

[72] K. K. Seth, "A new analysis of the $R$ measurements: Resonance parameters of the higher vector states of charmonium," arXiv:hep-ex/0405007 
[73] E. S. Ackleh, T. Barnes and E. S. Swanson, Phys. Rev. D 54, 6811 (1996) arXiv:hep-ph/9604355.

[74] T. E. Coan et al. [CLEO Collaboration], "First observation of $\psi(3770) \rightarrow \gamma \chi_{c 1} \rightarrow \gamma \gamma J / \psi$, arXiv:hep-ex/0509030

[75] E. Eichten, Phys. Rev. D 22, 1819 (1980).

[76] K. Heikkila, S. Ono and N. A. Tornqvist, Phys. Rev. D 29, 110 (1984) [Erratum-ibid. D 29, 2136 (1984)].

[77] N. Byers and E. Eichten, in EPS - high energy physics '89: proceedings, edited by F. Barreiro, et al., Nucl. Phys.
B (Proc. Suppl.) 16, 281 (1990).

[78] N. Byers, in Quark confinement and the hadron spectrum: proceedings, edited by N. Brambilla and G. M. Prosperi (World Scientific, Singapore, 1995), p. 139 arXiv:hep-ph/9412292.

[79] Xiaoyan Shen, "Heavy Flavor, Quarkonium Production and Decay," plenary lecture at Lepton / Photon 2005, Uppsala, http://lp2005.tsl.uu.se/ lp2005/LP2005/programme/presentati 